\section{Use of Paper Partition Chromatography in the Identification of the Volatile Fatty Acids}

THE volatile fatty acids are important in many biological processes. They may be separated, identified and estimated by means of partition chromatography using silica gel columns. The whole range from acetic to capric acids, inclusive, may be separated in this way; but this involves the use of more than one solvent ${ }^{1,2}$ (that is, separation in stages) or buffered columns ${ }^{3}$, and several titrations.

Lugg and Overell" separated organic acids as the free acids on paper sheets, using a mixture of butanol and acetic acid as the mobile phase, a method inapplicable, however, to the volatile fatty acids. Fink and Fink ${ }^{5}$ described a method in which the hydroxamates of the methyl esters of the volatile fatty acids were run on paper and developed with ferric chloride. Boldingh ${ }^{6}$ has run the ethyl esters of the fatty acids on paper strips coated with rubber and developed the spots with Sudan IV solution.

We have successfully used the method described below for the quick identification and rough estima. tion of the volatile fatty acids in culture fluids and cheese distillates. This method was based primarily on the silica gel technique of Lester Smith ${ }^{7}$ and Elsden, in that water was used as the stationary phase, butanol as the mobile phase and bromcresol-green as the indicator, which was sprayed on the paper as a 2 per cent solution in alcohol after the butanol had been driven off in a current of cool air.

The free fatty acids could not be used because of volatilization during the running of the chromatogram, neither could the sodium salts, because they remained stationary. Volatile weak alkalis were tried in the hope of controlling the ionization of the acids by varying the concentration of alkali, and although this was not achieved, satisfactory chromatograms were obtained by using ammonia or ethylamine in the vapour phase.

Chromatograms so prepared remained alkaline to brom-cresol-green even after an exposure of half an hour to the atmosphere; but on a further exposure of one to several hours the blue colour of the background gradually changed to greenish-yellow, while the spots representing the acids remained blue for at least several days. Eventually they, too, faded. We have not examined the reasons for this delayed development; but acidification by atmospheric carbon dioxide at a rate depending on the buffering power of the substances in the paper seems a plausible explanation.

The volatile fatty acids do not differ greatly in strength. For example, acetic, butyric and caproic acids were found to be completely titrated with ethylamine before the $p \mathrm{H}$ had risen to 6 . As there is an excess of the volatile alkali during chromatography and the $p \mathbf{H}$ is well above.6, the fatty acids in the aqueous phase will exist as anions. Thus the concentration of the alkali is not critical ; if the fatty acids are neutralized (to brom-cresol-green) with ethylamine before testing, a very low concentration of the vapour may be used, with the advantage that, on spraying, the blue spots develop at once on a bright yellow background and the contrast is greater than that obtained by allowing a blue chromatogram to fade. Brom-cresol-purple can replace brom-cresolgreen as developer, but the spots are less vivid. We have used ethylamine in preference to ammonia as the volatile alkali, since it gives more durable and more clearly defined spots. The spots are not perm.

\begin{tabular}{|c|c|c|}
\hline \multirow{2}{*}{ Fatty acids } & \multicolumn{2}{|c|}{$R_{F}$ values (room temperature) } \\
\hline (N/10) & Ammonia & Ethylamine \\
\hline Acetic & 0.11 & 0.20 \\
Propionic & 0.18 & 0.31 \\
Butyric & 0.28 & 0.44 \\
Valeric & 0.67 & 0.56 \\
Caproic & 0.67 & 0.77 \\
Caprylic & 0.81 & 0.91 \\
Capric & 0.87 & $=$ \\
\hline
\end{tabular}

anent, but the colour contrast in the fresh chromatograms is sufficiently good to give permanent photographic records. Typical $R_{F}$ values are shown in the accompanying table.

As with the amino-acids, the area of the spot gives a rough indication of the total quantity of fatty acid present ; $4 \mu 1$. of $N / 10$ solutions give convenient but rather large spots, $N / 100$ is a better concentration and $N / 1,000$ is just detectable.

Since this letter was written, a communication from F. Brown and L. P. Hall has appeared (Nature, July 8 , p. 66) describing a method which, though similar in some respects to the one we have described, gives yellow spots on a pale blue background. Presumably, the yellow spots indicate free acids liberated by the decomposition of the ammonium salts during the heating at $95^{\circ} \mathrm{C}$, primarily intended for the evaporation of the solvent. In our methed the solvent is allowed to evaporate at room tempera. ture and the ammonium or ethylamine salts do not decompose but, remaining in the water associated with the cellulose, give a solution alkaline to bromcresol-green, and therefore appear as blue spots on the chromatogram. The loss of volatile acids is thus reduced to a minimum, and the method gives roughly quantitative results.

E. R. Hrscox

N. J. BERRIDGE

National Institute for Research in Dairying, University of Reading. May 13.

${ }^{1}$ Ramsay, L. L., and Patterson, W. L., J. Assoc. Off. Agric. Chem., 31, 139, 441' (1948).

Peterson, M. M. H., and Johnson, M. J., J. Biol. Chem., 174, 775 (1948).

- Moyle, V., Baldwin, E., and Scarisbrick, R., Biochem.J., 43, 308 (1948). Lugg, J. W. H., and Overell, B. T., Nature, 160, 87 (1947).

s Fink, K., and Fink, R. M., Proc. Soc. Exp. Biol. and Med., 70, 654 (1949).

${ }^{6}$ Boldingh, J., Recueil, 69, 247 (1950).

? Lester Smith, E., Biochem. J., 36, Proc. xxii (1942); also private communication (1942).

${ }^{8}$ Elfden, S. R., Biochem. J., 40, 252 (1946).

\section{Differential Phase Change at Reflexion}

THE measurement of length by interferometry can only be made with the highest precision if corrections are applied for the differential phase changes produced at reflexion at the relevant surfaces. These corrections are very small, and their magnitude and relation to surface structure can best be studied by multiple-beam interferometry.

The phase change at reflexion at a surface appears to be a function of both the material and the surface finish. From measurements made on the height of steps etched in a polished glass surface, it was found that a small differential phase-change effect occurred due to the difference in surface roughness of the polished surface of the glass and the etched bottom of the step. For these measurements, the surface of 